Revista de Comunicación y Salud, 2018, Vol. 8, № 1, pp. 111-126

Editado por Cátedra de Comunicación y Salud

ISSN: 2173-1675

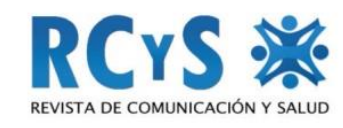

Enviado 17 de octubre de 2018

Aprobado 29 de noviembre de 2018

\title{
BLOG EN EDUCACIÓN ALIMENTARIA Y NUTRICIONAL. UNA HERRAMIENTA DE COMUNICACIÓN Y EDUCACIÓN PARA PROMOVER EN SALUD INTEGRAL
}

\author{
Blog in food and nutrition education. A communication and education tool to promote \\ integral health
Albino José Rojas Piñango ${ }^{1}$, Ernesto Elías De La Cruz Sánchez, Beatriz Elena Ramírez Hernández

Universidad Pedagógica Experimental Libertador. Venezuela

\section{Resumen}

Los blogs, con uso edu-comunicativo, se han extendido intensamente en la web en los últimos años, ya que permiten establecer una comunicación constante y directa entre los actores, a través de sus reflexiones, opiniones y comentarios, expresando sus inquietudes y temas de mayor interés. Un tema clave lo constituye la alimentación y nutrición en el campo de la formación docente en la Universidad Pedagógica Experimental Libertador, desde el año 2015 se ha desarrollado un blog relacionado con este tema (educacionan.blogspot.com). Él acompaña los procesos de investigación, docencia, extensión y servicio comunitarios en nuestra casa de estudio. El trabajo presenta una caracterización de la salud en Venezuela, la descripción de los blog como recurso didáctico y su construcción. Como resultado de su seguimiento se destaca: empleo de la herramienta Blogger-Google para su diseño, la publicación de 170 post, 100.000 visitas, 127 seguidores, la página más consultada es la referida a estrategias y recursos para la enseñanza de la educación alimentaria, la audiencia se ubica principalmente en USA, Venezuela, México y Colombia. El Open Site Explorer y Woorank, señalan su buena calidad, presentado: autoridad, objetividad, actualización, precisión de las fuentes, estético, buena navegabilidad, accesibilidad y posicionamiento en las redes sociales. Recomiendan suscribir a Facebook y Twitter. El blog se ha constituido en un recurso en línea idóneo para estudiantes y maestros en su formación permanente, sobre temas prioritarios en el campo de la salud, la alimentación y la nutrición.

Palabras clave: blog, comunicación, educación, salud, alimentación, nutrición, formación docente, pedagogía.

\section{Abstract}

Blogs, with they edu-communicative use, have been intensely spread on the web in recent years, as they allow establishing a constant and direct communication

\footnotetext{
${ }^{1}$ Autor para correspondencia: Albino José Rojas Piñango arojas 5@hotmail.com
} 
Blog en educación alimentaria y nutricional. Una herramienta de comunicación y educación para promover en salud integral

between the actors, through their reflections, opinions and comments, expressing their concerns and issues of major interest. A key issue is food and nutrition in the field of teacher training at the Universidad Pedagógica Experimental Libertador, for which a blog has been developed since 2015. (educacionan.blogspot.com). It accompanies the research, teaching, extension and community service processes of our students. The work presents a description of the blog as a teaching resource and the characterization of health in Venezuela and the construction of this proposal. As a result of its follow-up, it stands out: it used the Blogger-Google tool for its design, the publication of 170 posts, 100,000 visits, 127 followers, the most consulted page refers to strategies and resources for teaching food education, The audience is located mainly in the USA, Venezuela, Mexico and Colombia. The Open Site Explorer and Woorank, indicate its good quality, presented authority, objectivity, updating, accuracy of sources, aesthetics, good navigability, accessibility and positioning in social networks. They recommend subscribing to Facebook and Twitter. Being an ideal online resource for students and teachers in their permanent training, on priority issues in the field of food and nutrition.

Keywords: blog, communication, health, food, nutrition, teacher training, education, pedagogy.

\section{Cómo citar el artículo}

Rojas Piñango, A. J.; De La Cruz Sánchez, E. E. y Ramírez Hernández, B. E. (2018). Blog en educación alimentaria y nutricional. Una herramienta de comunicación y educación para promover en salud integral. Revista de Comunicación y Salud, 8(1), pp. 111-126.

DOI: http://doi.org/10.35669/revistadecomunicacionysalud.2018.8(1).111-126

\section{EL BLOG COMO RECURSO COMUNICACIONAL Y DIDÁCTICO}

En 1994, Justin Hall, estudiante de la Universidad de Swarthmore, crea Link.net, el cual se dedicaba a comunicar aspectos personales y tenía algunas secciones de noticias. Luego Dave Winer aparece en la red con Scripting News en 1997 hablando de tecnología y política. Dave ofreció contenido valioso para sus seguidores, no sobre su vida, sino de opiniones y novedades, además de tener el diseño actual de los blogs. Es en 1997, cuando Jorn Barger de Robotwisdom.com crea la palabra weblog, que proviene de la contracción de las palabras "web log" del idioma inglés, en español significa bitácora web.

Ahora es el popular blog; proveniente de la deformación de la palabra weblog dividida por Peter Merholz en we blog haciendo referencia a publicaciones en línea de historias; desde su llegada ha estado a la disposición de todas las áreas disciplinarias.

Sus comienzos como recurso para el anuncio y compartir reflexiones con los usuarios de ciertas empresas, ha tomado popularidad en la publicidad, economía, las ciencias, la salud y sobre todo en la educación. En esta última se utiliza como uno de los recursos fundamentales para la enseñanza y el aprendizaje en cualquier nivel y modalidad educativa, así como en la capacitación y actualización de los 
Blog en educación alimentaria y nutricional. Una herramienta de comunicación y educación para promover en salud integral

docentes y público en general; ya que permite el desarrollo de competencias en el proceso de aprendizaje, tal como lo refiere Parada (2014):

...comprensión lectora, integración de diversas fuentes de información, práctica de la escritura en diferentes contextos sociales y distintos géneros y formatos, integración de textos junto a elementos gráficos y multimedia, ya que es una de las herramientas de la tecnología, más flexibles y potente, para organizar y publicar contenido de diversos ámbitos disciplinares e integrar recursos procedentes de los servicios de la Web 2.0 (p. 33).

Es por ello que los blogs con uso educativo permiten establecer una comunicación constante y directa entre los actores de la comunidad educativa y su creador, a través de las reflexiones, cambios de opiniones y comentarios. Es tan versátil que las estrategias y la información breve despiertan el interés del lector llevándolo a investigar y motivando a la búsqueda y desarrollo de las ideas expuestas por el autor o por el lector.

El diseño de un blog es fácil, sin complicaciones o conocimientos técnicos avanzados, su mantenimiento y modificación no es complicada, porque los creadores de este recurso en la Web han propuestos plantillas clasificadas según su objetivo; así como dando la oportunidad a los usuarios o lectores puedan emitir comentarios. Esta interactividad, además de la facilidad para su creación y administración, es lo que ha influido en su incremento dentro de la Internet. Es por eso que el docente encuentra en él un recurso en línea para publicar sus contenidos en una forma atractiva y de fácil manejo, apoyándose en audio, vídeo, animaciones, presentaciones, documentos, infografías, encuestas, actividades interactivas, entre otros. (Palacio, 2016).

La aceptación de los estudiantes y público interesado en los temas tratados, depende de las actualizaciones de los contenidos, la versatilidad, profundidad, el uso de diferentes recursos y permanencia en el tiempo está relacionada con la creatividad y la constancia del docente.

El blog constituye uno de los tantos recursos clasificados como la Web 2.0 o Web social; la cual se entenderá como aquellos sitios web que facilitan: compartir información, la interoperabilidad, el diseño centrado en el usuario y la colaboración en la World Wide Web (www) de manera que interactúa con una comunidad virtual, siendo uno de los medios para comunicar, expresar, opinar, construir conocimiento, compartir contenidos y obtener información. Se pueden desarrollar como sitios independientes, o en secciones o anexos del sitio web principal. En la actualidad además existen los blogs que se asemejan a portales informativos, con noticias y artículos periodísticos, donde su énfasis es la comunicación e información.

Los docentes a la hora de diseñar un blog para impartir conocimientos es necesario entender cuáles son sus características; en general, Mejía (2016), y Palacios (2016), coinciden en destacar:

- Es una plataforma accesible.

- El contenido se dividen en posts -artículos breves- que se desplazan de manera cronológica cada vez que se publica uno nuevo. 
- Es flexible por ser un software avanzado que permite el acceso a contenidos digitales.

- Las páginas son estáticas.

- Los lectores suelen tener la opción de comentar el artículo publicado, necesario en el ámbito educativo, creándose así una relación bidireccional entre el autor y sus lectores.

- En un post se pueden utilizar textos, enlaces, imágenes, vídeos, audios, animaciones, infografías, entre otros recursos.

- Utiliza etiquetas y categorías para facilitar la búsqueda de contenidos dentro del blog.

- En el contenido de un blog se pueden utilizar palabras clave seleccionadas para conseguir posicionar el blog y recibir así tráfico web.

- Un blog normalmente permite suscribirse a él con la dirección de email para recibir actualizaciones.

- Facilita pantallas más agradables y fáciles de usar.

- Las personas acceden a la Web, crean la Web y le dan popularidad, generando un proceso socializador y un movimiento gradual hacia el mundo en línea.

- Es un flujo de sucesos y conversaciones, donde lo importante es la conectividad y, los usuarios son vistos como codesarrolladores.

- Son una excelente herramienta que combina aspectos comunicativos, educativos, sociales y motivadores.

Por lo tanto, la estructura de los blogs los convierte en una herramienta útil para el uso en la enseñanza donde se destacan: (a) Sistema de almacenamiento de la información; (b) El rol del docente deja de ser un mero transmisor de conocimientos y se convierte en guía, curador de contenidos y prosumidor de información (abandona la faceta pasiva para convertirse en generador de contenidos y creador de ideas y opiniones que ejercen influencia a la comunidad); y el estudiante muestra sus progresos en una determinada actividad o asignatura, expone sus ideas $u$ opiniones, diario de trabajo realizado, entre otras actividades; (c) Gestor a través de la Internet, se puede acceder en cualquier momento y lugar, con un computador conectado a la red; (d) Trabaja ciertos aprendizajes curriculares pero también se obtienen nuevos aprendizajes no formales, y facilita la retroalimentación crítica como consecuencia de los comentarios por parte de los lectores, que pueden provenir de los docentes, compañeros o de una amplia audiencia (Rojas, 2016).

La facilidad con que se crean y alimentan los blog, los hace muy llamativos porque gracias a los asistentes y las plantillas prediseñadas, hacen que el docente no se concentre y agote en la implementación técnica sino en los contenidos y materiales. Por lo que el uso educativo del blog se destaca por: 


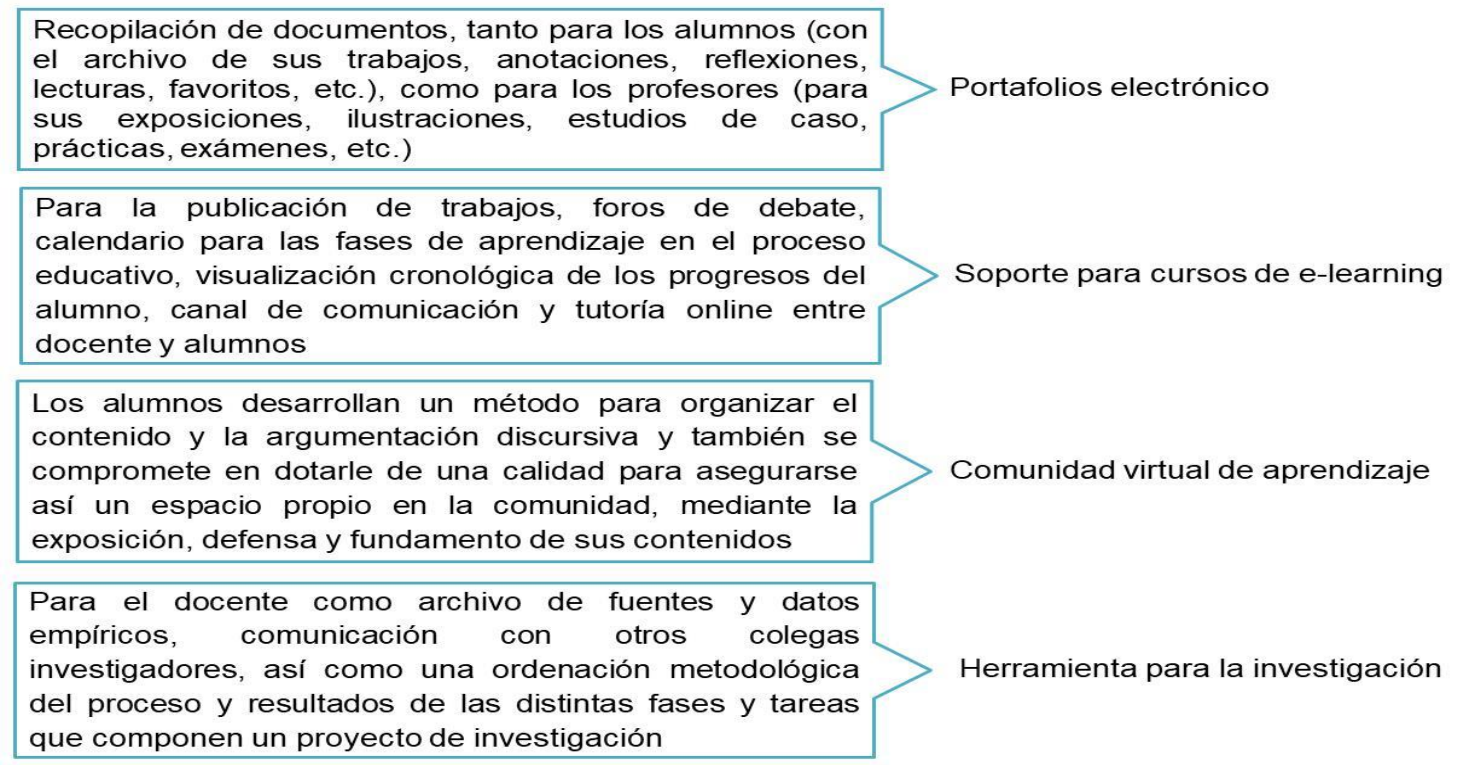

Figura 1. Posibilidades educativas del blog. Tomado de Gabinete de Tele-Educación. Universidad Politécnica de Madrid, 2014, p. 95.

Según Osorio (2016), el blog es uno de los fenómenos más exitosos de Internet, lastimosamente no es tan destacado y no recibe el reconocimiento como una herramienta útil para compartir, recibir información y para comunicarse en la red. Es un excelente método para publicar y dar a conocer conocimientos de una forma pública y para que otras personas se beneficien de los temas tratados en este sitio.

Estas posibilidades se las brindan los diversos programas, aplicaciones, software o paquetes utilizados para la creación o diseño de blog, lo más recomendados son aquellos que se ofrecen de forma gratuita en Internet, ya que no son necesarios conocimientos informáticos avanzados, es rápido y de fácil uso. Las aplicaciones gratuitas son:

Weebly: Es muy fácil de utilizar, se pueden ubicar varios blog en una misma página web, cargar infinidad de widgets, permite crear cuestionarios, encuestas. Y luego ver los resultados de los mismos desde su aplicación para el teléfono. Al principio parece muy sencilla pero combinando bien sus posibilidades se consigue hacerla una herramienta ideal. Otra cosa interesante es su versión para MAESTROS, ofrece una variedad de propósitos, algunos de los cuales incluyen: crear un sitio web para el aula, diseñar portafolios electrónicos para estudiantes y crear sitios web par a proyectos asignados. https://www.weebly.com/ve

Blogger: El más utilizado por todos, este es el que te ofrece Google, por lo tanto si se tiene un correo Gmail, simplemente se ingresa y empieza a crear, Muy fácil e intuitivo, estable y goza de toda tu comunidad google+. https://www.blogger.com

WordPress: De los más profesionales en su versión de pago, algo complejo de utilizar si no tienes mucha idea. Es el sistema de gestión de
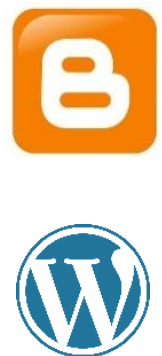

Revista de Comunicación y Salud, 2018, Vol. 8, no 1, pp. 111-126 
Blog en educación alimentaria y nutricional. Una herramienta de comunicación y educación para promover en salud integral

contenido para la creación de blogs que más desarrollo está teniendo en los últimos tiempos. Altamente personalizable con una gran variedad de plugins y diseños. Es muy fácil de instalar y de usar. http://wordpress.org.

Edublogs: Es un servicio para que docentes y estudiantes publiquen de forma gratuita su blog. El sistema utiliza WordPress con casi todas las ventajas y funcionalidades de otros blogs gestionados con esta aplicación. Tiene el inconveniente de que el proceso de la gestión del blog es en idioma inglés. http://edublogs.org

Bitácoras: Está presente en la Internet desde el año 2002 y es el mayor centro de servicios y promoción de blogs en español, así como una red social para blogueros. El sistema incorpora un filtro social que recoge la información de miles de blogs, y la presenta de forma organizada y catalogada pudiendo ser valorada por los usuarios. http://www.bitacoras.com

Dichas herramientas informáticas, presentan grandes posibilidades de ser empleadas en diversos campos de interés para las colectividades. Uno de ellos, de gran valor y relevancia en la actual situación país y en especial dentro de la formación docente en la Universidad Pedagógica Experimental Libertador, lo constituye el campo de la salud integral y en especial la educación alimentaria y nutricional.

\section{LA ALIMENTACIÓN Y LA NUTRICIÓN EN EL CONTEXTO DE LA SALUD INTEGRAL}

En este contexto general, la Organización Mundial de la Salud en su Carta Magna de 1946, define la salud como "Completo estado de bienestar físico, psíquico y social y, no solamente la ausencia de afecciones o enfermedades". Esto nos lleva a entender a la salud tanto como un hecho social como inmaterial. Por lo tanto, se trata de un fenómeno multidimensional y multicausal que trasciende y desborda la competencia estrictamente médica, relacionándose además la biología y la psicología, con la economía, la cultura, el ambiente y la política, en tanto que es un asunto individual y colectivo al unísono, (Alcántara, 2008). Estos referentes nos aproximan al concepto actual de salud situándonos en un plano integrador con la sociedad y el hombre, que podríamos referir como: "Conjunto de condiciones físicas, psíquicas y sociales que permiten a la persona desarrollar y ejercer todas sus facultades en armonía y relación con su propio entorno" (Perea, 2002, p.12). En este escenario los procesos edu-comunicativos cobran especial relevancia.

Razón por la cual hay que reflexionar sobre las variables que actúan sobre la salud, Sainz, López y van den Boom (2001), destacan los cuatro factores fundamentales que actúan sobre la salud: Los genéticos o biológicos (14\%), los sanitarios $(20 \%)$, los medio-ambientales (33\%) y los estilos de vida (33\%). En esta visión los factores ambientales y los estilos de vida son los elementos determinantes de la salud y sobre todo este último, está vinculado particularmente a la educación.

Situación que se expresa actualmente para Venezuela, en altos niveles de malnutrición (del exceso al déficit) [INN, 2013, ENCOVI, 2015-2016], incremento en 
Blog en educación alimentaria y nutricional. Una herramienta de comunicación y educación para promover en salud integral

las tasas de mortalidad asociadas a enfermedades no transmisibles, las cuales constituyen evidencias cada vez más frecuentes de este fenómeno. En nuestro caso, es importante destacar a las enfermedades crónicas no transmisibles (ECNT), dentro de las principales causas de mortalidad, registradas y diagnosticadas, desde el año 2005 al 2013 (último año de reporte MPPS), donde se ubican a las enfermedades del corazón, cáncer, diabetes y enfermedades cerebrovasculares. Adicionalmente se destaca el aumento en la diabetes como causa de muerte (Ministerio del Poder Popular para la Salud, 2015). Lo que expresa, que en Venezuela existe una alta prevalencia de mortalidad asociada a ECNT, las cuales están fuertemente asociadas a hábitos de salud, estilos de vida y dentro de ellos los de alimentación y nutrición.

\section{Cuadro 1.}

Principales causas de mortalidad para la República Bolivariana de Venezuela.

\begin{tabular}{|c|c|c|c|c|c|}
\hline $\begin{array}{l}\text { Año } \\
\text { Número de defunciones } \\
\text { Causa de Mortalidad }\end{array}$ & $\begin{array}{c}(2005) \\
117.831\end{array}$ & $\begin{array}{c}(2007) \\
127.436\end{array}$ & $\begin{array}{c}(2009) \\
134.731\end{array}$ & $\begin{array}{c}(2011) \\
143.020\end{array}$ & $\begin{array}{c}(2013) \\
149.903\end{array}$ \\
\hline Enfermedades del corazón & $20,6 \%\left(1^{\circ}\right)$ & $20,0 \%\left(1^{\circ}\right)$ & $20,3 \%\left(1^{\circ}\right)$ & $21,3 \%\left(1^{\circ}\right)$ & $20,6 \%\left(1^{\circ}\right)$ \\
\hline Cáncer & $15,4 \%\left(2^{\circ}\right)$ & $15,1 \%\left(2^{\circ}\right)$ & $15,0 \%\left(2^{\circ}\right)$ & $15,6 \%\left(2^{\circ}\right)$ & $15,4 \%\left(2^{\circ}\right)$ \\
\hline $\begin{array}{l}\text { Enfermedades cerebro- } \\
\text { vasculares }\end{array}$ & $7,3 \%\left(3^{\circ}\right)$ & $7,6 \%\left(3^{\circ}\right)$ & $7,5 \%\left(3^{\circ}\right)$ & $7,7 \%\left(3^{\circ}\right)$ & $7,4 \%\left(4^{\circ}\right)$ \\
\hline Diabetes & $6,2 \%\left(6^{\circ}\right)$ & $6,1 \%\left(6^{\circ}\right)$ & $6,5 \%\left(6^{\circ}\right)$ & $6,8 \%\left(4^{\circ}\right)$ & $7,6 \%\left(3^{\circ}\right)$ \\
\hline Total & $49,5 \%$ & $48,8 \%$ & $49,3 \%$ & $51,4 \%$ & $51,0 \%$ \\
\hline
\end{tabular}

Fuente: Anuarios de Mortalidad, Ministerio del Poder Popular para la Salud (2005-2015).

Nota: El número entre paréntesis expresa la posición relativa de la causa de mortalidad. Las otras causas de mortalidad se reportan por homicidios-suicidios y accidentes de tránsito.

En, un contexto más específico, se destaca la Estrategia mundial sobre régimen alimentario, actividad física y salud (OMS, 2004). La estrategia aborda dos de los principales factores de riesgo de las ECNT, el régimen alimentario y la actividad física, parte del reconocimiento y preocupación por el problema y su impacto a nivel económico, social y cultural; de lo cual se propone: (a) reducir los factores de riesgo, (b) promover la conciencia y el conocimiento en la población en torno al potencial positivo de una alimentación saludable y la actividad física; (c) establecimiento y fortalecimiento de políticas y planes de acción en este sentido; y (d) el seguimiento, monitoreo, la investigación científica y la evaluación de las intervenciones para mejorar futuras acciones.

En forma particular, la educación alimentaria y nutricional promueve mejoras en los conocimientos y actitudes de las personas para una calidad de vida más sana, mediante el establecimiento de conductas acorde con las necesidades del individuo y con el objetivo central de mejorarlo y reforzarlo de forma positiva con el paso del tiempo.

La Fundación Bengoa (2010), plantea que la salud y el buen funcionamiento de nuestro organismo, dependen de la nutrición y alimentación que se tenga durante la

Revista de Comunicación y Salud, 2018, Vol. 8, nº 1, pp. 111-126 
vida. Aun cuando parecieran significar lo mismo, son conceptos diferentes: La Nutrición es el conjunto de procesos que permiten que nuestro organismo utilice los nutrientes que contienen los alimentos para realizar sus funciones. Los alimentos, son todos los productos naturales o industrializados que consumimos para cubrir una necesidad fisiológica (hambre) y la alimentación permite tomar del medio que nos rodea, los alimentos de la dieta. Razón por la cual las actitudes, factores socio-económicos, culturales y edu-comunicativos tienen enorme relevancia en la consolidación de hábitos saludables de alimentación. También es necesario aproximarse a entender a la alimentación como un hecho bio-psico-social complejo y como tal hay que abordarlo: la gramática culinaria, las categorizaciones de los diferentes alimentos, los principios de exclusión y de asociación entre tal y cual alimento, las prescripciones y las prohibiciones tradicionales y/o religiosas, los ritos de la mesa y de la cocina, etc., son todo ello estructura de la alimentación cotidiana (De La Cruz, 2013 y 2017).

En la actualidad, la conducta alimentaria está determinada por diversos factores, algunos de los cuales no se relacionan directamente con los alimentos: sociales, económicos, publicitarios, culturales, etc. Este hecho, muestra la relevancia en el proceso de educación nutricional de la sociedad, ofreciendo una alimentación equilibrada y proporcionando información real acerca de los diferentes alimentos. Una correcta educación nutricional podría conseguir en la sociedad actual buenos hábitos alimentarios que actúen como factores de prevención de enfermedades cardiovasculares, obesidad, diabetes o diferentes tipos de enfermedades (FAO, 2014).

En tal sentido, es importante considerar que los hábitos alimentarios y el estilo de vida saludable se asimilan e integran a la personalidad durante los primeros años hasta la edad adulta; de ahí la importancia de brindar una orientación adecuada por parte de los docentes y la familia, responsables de promover la enseñanza y el aprendizaje de hábitos saludables y valorarlos como herramientas que ayuden a proteger y cuidar la salud. Para el Instituto Nacional de Nutrición (2008), la educación nutricional y los hábitos saludables ayudan a sensibilizar sobre las costumbres, actitudes, formas de comportamientos que asumen las personas ante situaciones concretas de la vida diaria, las cuales conllevan a formar y consolidar pautas de conducta y aprendizajes que se mantienen en el tiempo y repercuten (favorable o desfavorablemente) en el estado de salud y el bienestar mediante los hábitos que se aprende gradualmente por imitación.

En síntesis, la alimentación es una de las acciones, de mayor importancia, que afecta el bienestar de las personas de modo considerable. Una buena dieta puede ayudar a mantener la salud e incluso hasta mejorarla, al escoger alimentos variados y consumirlos con moderación. La alimentación de los individuos depende en gran parte del poder adquisitivo, pero sobre todo de la información que posea la persona sobre cantidad-calidad de alimentos, de los hábitos alimentarios, de las tradiciones gastronómicas que tenga y como intervenir para mejorarlos, inclusive los asociados a su cultura. Aquí los recursos tecnológicos, TIC, web, en especial los blog con estos enfoque contribuyen de manera significativa a informar, educar, formar y proponer alternativas más saludables que contribuyan a mejorar la calidad de vida presente y futura, con énfasis en la prioridad que debe tener la formación docente. 


\section{EL BLOG EN EDUCACIÓN ALIMENTARIA Y NUTRICIONAL, UN CAMINO}

En el contexto específico la UPEL, la cual se define como: una universidad pública de alcance nacional e internacional; líder en la formación, capacitación, perfeccionamiento y actualización de docentes de elevada calidad personal y profesional; en la producción y difusión de conocimientos socialmente válidos, que desarrolla procesos educativos con un personal competente y comprometido con los valores que promueve la Universidad. Su Visión se orienta a la generación de conocimientos útiles para implementar procesos pedagógicos innovadores e impulsar la transformación de la realidad social y así contribuir al logro de una sociedad más próspera, equitativa y solidaria.

Para la consolidación de esta visión se requiere que el maestro, desde la escuela, se abra a nuevas experiencias, que actualice su repertorio pedagógico logrando así poder transformar el hecho educativo desde los primeros niveles de escolaridad y que logre un impacto trascendente para la verdadera inserción social y desarrollo integral del individuo, la participación y compromiso de la comunidad con dichos fines en términos de sus capacidades y aptitudes para la convivencia, el respeto, la tolerancia, autorrealización personal, profesional y laboral.

A la par de la educación para la salud, la Educación Alimentaria y Nutricional constituye una propuesta estratégica, en la formación humana para la vida. Es una línea de trabajo pedagógico que nos permite adquirir mayor consciencia acerca de la importancia que le debemos otorgar a la primera necesidad humana y nos induce a revalorar la propia cultura alimentaria. Por ello, la educación en alimentación y nutrición debe orientarse a potenciar o modificar los hábitos alimentarios, involucrando a todos los miembros de la comunidad educativa; niños, padres, maestros y directivos. Educar sobre la necesidad e importancia de una buena y/o adecuada alimentación implica: descubrir y erradicar creencias, mitos y conductas erróneas; promoviendo una mayor consciencia sobre las diversas funciones o roles que juega o debe jugar la alimentación en las diversas esferas de la vida, la salud, los aprendizajes, la producción, manipulación, distribución y consumo de alimentos; fomentar conceptos, actitudes y conductas claras y fundamentales sobre la alimentación (De La Cruz, 2008).

Razón por la cual, el aportar nuevas alternativas de comunicación, formación e información en materia de alimentación y nutrición, se constituye en un recurso didáctico significativo. Este escenario, en los actuales momentos que vive nuestro país con escasos recursos documentales, limitado acceso a la información y recesión económica. Puede generar un proceso de resonancia edu-comunicacional que trascienda lo unidireccional y se aboque a repercutir a nivel de la familia, la escuela y la comunidad. 


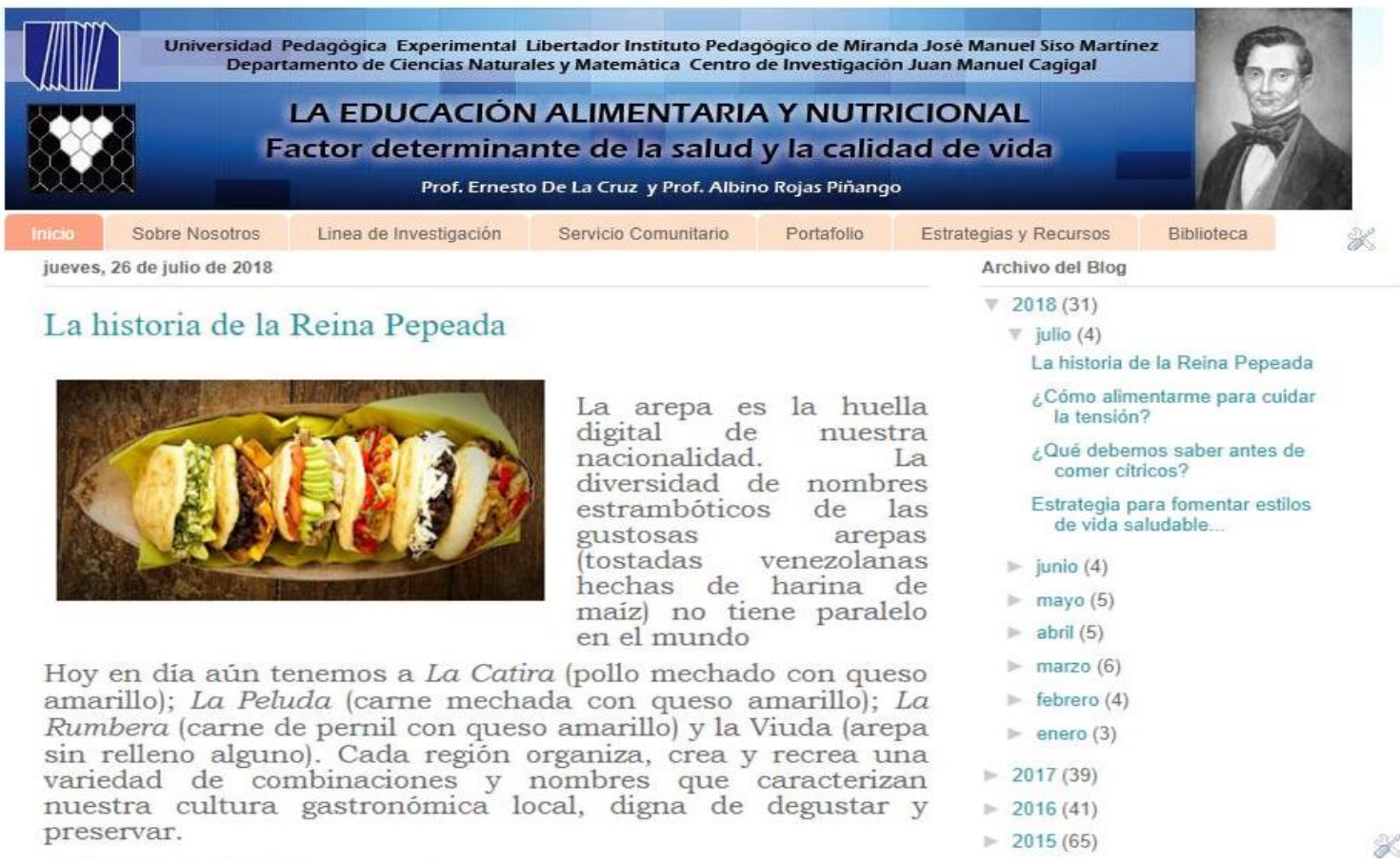

Figura 2. Captura de pantalla del blog en educación alimentaria y nutricional. http://educacionan.blogspot.com. 26 de julio 2018.

Fuente: autores.

Para ello, el blog en educación alimentaria y nutricional: (a) aporta aspectos en la formación docente con énfasis en las especialidades de educación preescolar y primaria para el sistema educativo venezolano; (b) sirve como apoyo al programa de servicio comunitario desde el año 2015; (c) mejora la visibilidad de nuestros productos y proyectos inscritos en la línea de investigación, (d), subsana las limitaciones conceptuales, procedimentales y actitudinales en el campo de la alimentación y nutrición que evidencian nuestra población estudiantil, (e) presenta recursos, estrategias y actividades en torno al tema, y (f) facilita materiales documentales, bibliográficos actualizados y pertinentes a su formación profesional.

El mismo se diseña bajo el recurso Bloggers de Google, por su fácil manejo y administración, amplitud de recursos multimedios (imágenes, fotos, textos, videos). En el cual se presentan lecturas-post-artículos de diferentes temáticas de interés. Se estructura en una serie de "pestañas" con información de los investigadores, aspectos de interés en el proyecto de servicio comunitario, las áreas temáticas asumidas por la línea de investigación, una descripción de los productos (portafolio) que se han generado durante estos años, las estrategias y recursos propuestos por los propios estudiantes e investigadores para la enseñanza de la alimentación y nutrición infantil, y la biblioteca.

Estos escenarios constituyen elementos de relevancia y pertinencia para nuestros estudiantes de pregrado y postgrado de la UPEL, investigadores de otras áreas y disciplinas inter-extra institucionales, y la comunidad en general al interesarse por la, capacitación, actualización, asesoría (extensión). 
Blog en educación alimentaria y nutricional. Una herramienta de comunicación y educación para promover en salud integral

\section{LOGROS ALCANZADOS. UNA MIRADA AL CAMINO}

En este punto, se presentan las metas conquistadas durante estos tres años 2015-2018: artículos publicados, la expansión de nuestra audiencia y visitas a nuestro blog, páginas aliadas, y niveles de calidad alcanzados y señalados por recursos especializadas en estos productos multimedios.

Las visitas a nuestro blog alcanzan para julio 2018 las 100.000. Estableciéndose un comportamiento de 100 visitas diarias en promedio, reflejando el interés moderado a nivel nacional e internacional de nuestra presencia en las redes sociales, (ver figura 3). En el caso venezolano, se han visto reducidas las visitas debido a las dificultades de conectividad que presenta la población en general y que limitan su acceso, fortaleciendo el ingreso por vía telefónica.

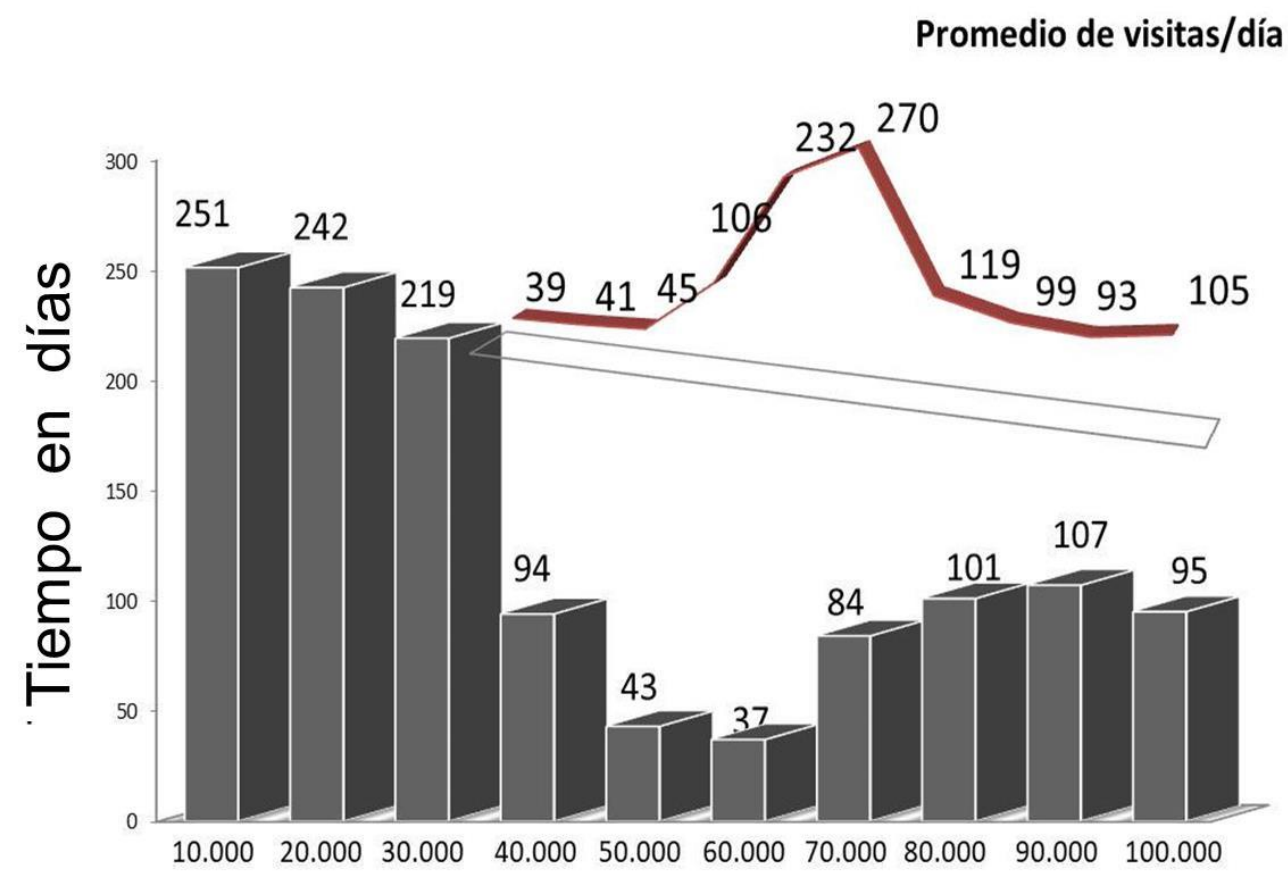

Figura 3. Tiempo en días para alcanzar 100.000 visitas entre el 13 de marzo 2015 y el 20 de junio de 2018. Promedio de visitas diaria para el mismo intervalo de tiempo.

Fuente: autores.

Por otra parte al revisar la fuente nacional-internacional de nuestra audiencia es interesante evidenciar en que países se encuentran nuestros lectores, en las cuales se destacan que el $58,1 \%$ de los visitantes se distribuyen en 4 países (Estados Unidos, Venezuela, México y Colombia).

Esto se ha reforzado por la regularidad y constancia en la cual se han publicado los artículo o post, entre marzo 2015 y julio 2018, en dicho lapso se han editado un total de 170 artículos (1 artículo por semana en promedio). 
Blog en educación alimentaria y nutricional. Una herramienta de comunicación y educación para promover en salud integral

\begin{tabular}{|l|c|}
\hline Estados Unidos & $19,5 \%$ \\
\hline Venezuela & $19,4 \%$ \\
\hline México & $12,4 \%$ \\
\hline Colombia & $6,8 \%$ \\
\hline Rusia & $5,6 \%$ \\
\hline Perú & $5,2 \%$ \\
\hline España & $4,5 \%$ \\
\hline Argentina & $3,9 \%$ \\
\hline Ecuador & $2,9 \%$ \\
\hline Chile & $1,5 \%$ \\
\hline \multicolumn{1}{|c|}{ TOTAL } & $\mathbf{8 1 , 7 \%}$ \\
\hline
\end{tabular}

Figura 4. Distribución porcentual de las visitas a las lecturas entre marzo 2015 y julio 2018 (170 lecturas a la fecha). 100.000 visitas al blog.

Fuente: Blogger-Google-Estadísticas.

En el siguiente cuadro examinamos cuales han sido los 10 temas -post- que más han sido consultados por nuestra audiencia en estos tres años.

\section{Cuadro 2.}

Porcentaje de las visitas por tema o lectura durante el periodo marzo 2015-junio 2018. Total de lecturas. 170, total de visitas 100.000. Entre marzo 2015 y julio 2018. educacionan.blogspot.com

\begin{tabular}{|c|c|}
\hline Lectura-artículo-post & Porcentaje \\
\hline $\begin{array}{l}\text { La familia, la escuela y la comunidad son corresponsables en la educación } \\
\text { nutricional. } \\
\text { Publicado el } 30 \text { junio } 2015\end{array}$ & $2,1 \%$ \\
\hline $\begin{array}{l}\text { Hacia una pedagogía de la Educación Alimentaria y Nutricional } \\
\text { Publicado el } 2 \text { julio } 2015\end{array}$ & $1,8 \%$ \\
\hline $\begin{array}{l}\text { Historia de la educación Nutricional en Venezuela. } \\
\text { Publicado el } 21 \text { de abril de } 2015\end{array}$ & $1,4 \%$ \\
\hline $\begin{array}{l}\text { Pautas estratégicas para la alimentación y nutrición. } \\
\text { Publicado el } 09 \text { de junio de } 2016\end{array}$ & $1,2 \%$ \\
\hline $\begin{array}{l}\text { Bases fisiológicas para la alimentación complementaria. } \\
\text { Publicado el } 26 \text { de mayo de } 2016\end{array}$ & $0,6 \%$ \\
\hline $\begin{array}{l}\text { La Educación alimentaria y nutricional desde una dimensión sociocultural. } \\
\text { Publicado el } 09 \text { de febrero de } 2017\end{array}$ & $0,5 \%$ \\
\hline $\begin{array}{l}\text { Libro de actividades del día mundial de la Alimentación (FAO). } \\
\text { Publicado el } 13 \text { de octubre de } 2016\end{array}$ & $0,5 \%$ \\
\hline $\begin{array}{l}\text { Estatus de deficiencias de micronutrientes en el niño. } \\
\text { Publicado el } 01 \text { de diciembre de } 2016\end{array}$ & $0,5 \%$ \\
\hline $\begin{array}{l}\text { OMS. Debemos acabar con la obesidad infantil } \\
\text { Publicado el } 20 \text { de octubre de } 2016\end{array}$ & $0,4 \%$ \\
\hline $\begin{array}{l}\text { Efectos de la desnutrición temprana en el desarrollo y aprendizaje del niño } \\
\text { Publicado el } 21 \text { de julio de } 2015\end{array}$ & $0,4 \%$ \\
\hline $\begin{array}{r}\text { TOTAL: } \\
\end{array}$ & $9,4 \%$ \\
\hline
\end{tabular}

Fuente: autores.

Se observa en el cuadro, una distribución porcentual baja en estas 10 primeras lecturas $(9,4 \%$ en total), lo que pudiera indicar un interés homogéneo por la gama y variedad de artículos publicados y los cuales cubren aspectos: educativos, 
Blog en educación alimentaria y nutricional. Una herramienta de comunicación y educación para promover en salud integral

culturales, de salud integral, recomendaciones para padres, maestros, comunidad. Políticas públicas, nutrición comunitaria, ECNT, malnutrición, entre otros. Adicionalmente, como estrategia comunicativa, cada seis meses se publica el ranking (Top 25) de los artículos o post más visitados "temas de interés", esto permite al visitante tener una panorámica de las lecturas y la diversidad de temas que puede encontrar.

El blog presenta una serie de pestañas (páginas) con información estructural: sobre nosotros (los autores), la caracterización de la línea de investigación, el programa de servicio comunitario, portafolio (nuestros libros, publicaciones y ponencias), estrategias y recursos y la biblioteca.

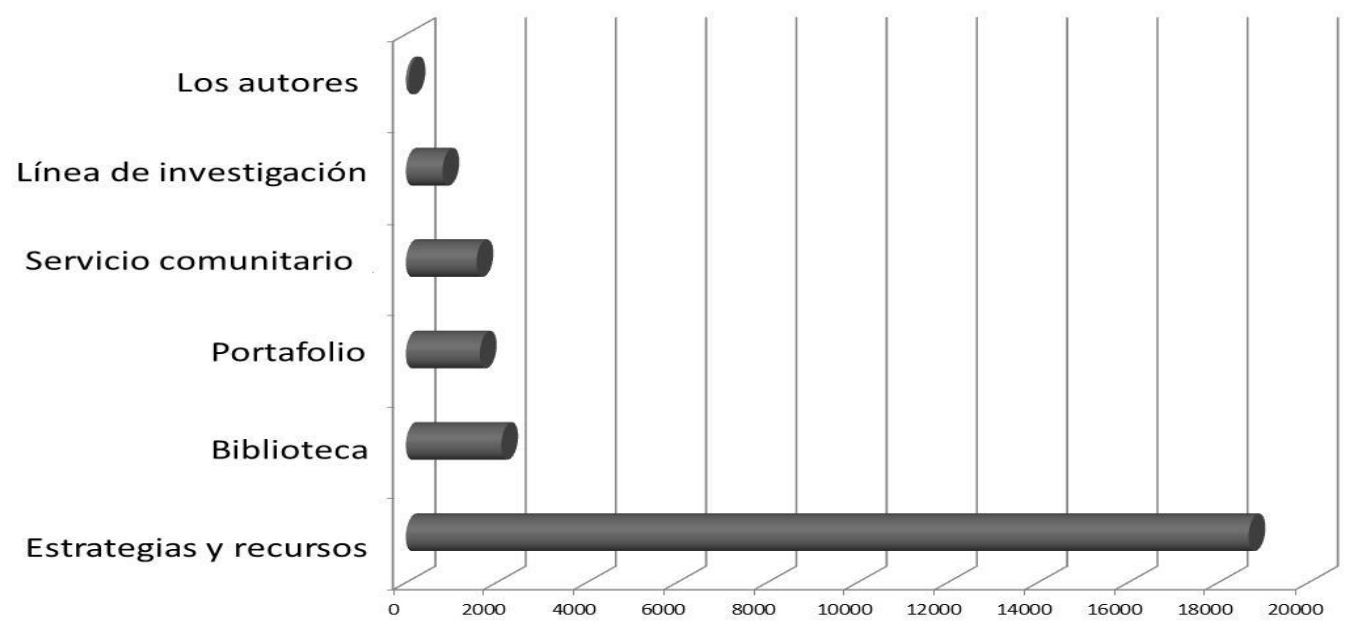

Figura 5. Frecuencia en la visita a las pestañas del blog. educacionan.blogspot.com. marzo 2015-julio 2018.

Fuente: autores.

Se observa en la figura, el gran interés que tienen nuestros lectores por las propuestas estratégicas (41 actividades publicadas), referidas a la enseñanza del tema de alimentación y nutrición, con énfasis en la educación infantil, con actividades lúdicas, creativas, vinculadas con la literatura, matemática, ciencias naturales, entre otras. Este recurso es consultado por educadores, estudiantes de nutrición-dietética, padres y representante, quienes han comentado el valor de este espacio.

Por otra parte, el ingreso al blog es fundamentalmente a través de los navegadores: Chrome (72\%), Internet Explorer (11\%), Firefox (10\%), Safari (2\%). El sistema operativo más frecuentemente empleado: Windows (63\%), Macintosh (16\%), Android (13\%), Linux (2\%), IPhone (1\%).

Al evaluar nuestro recurso, encontramos un variado número de criterios e indicadores para mejorar la propuesta edu-comunicativa. Los expertos señalan que existen más de 1.000 millones de sitios web en línea, y sigue aumentando aceleradamente. El $40-50 \%$ de la población mundial tiene acceso a internet y la utilizan mayoritariamente para buscar información y para comunicarse con otras 
personas. Casi el 75\% de la información que existe en internet está escrita en blogs, sitios web de personas naturales o son proyectos personales.

Razón por la cual se requiere, para su mejoramiento constante, tener presente una serie de aspectos para evaluar estos recursos dentro de los que se destacan: autoridad, actualización, navegabilidad, organización, selección y precisión de los contenidos, objetividad, legibilidad y adecuación al destinatario. Para ello existen recursos en línea que nos permite tener una visión comparada del blog con los criterios señalados, entre ellos destacamos: Open Site Explorer (Moz.com) y Woorank (herramienta SEO), por mantener claridad en estos criterios, de fácil comprensión y de naturaleza gratuita, entre otros.

Los resultados nos evidencian: $88 \%$ de dominio y autoridad. $55 \%$ de calidad, expresando una buena presencia en redes sociales, con evidencia de autoridad, constante actualización, con fuentes confiables obtenidas de revistas científicas de alta confiabilidad, precisión de los datos y comprobable.

El blog posee muy buena navegabilidad, legibilidad y estética en la presencia de sus recursos; sus contenidos son objetivos, la temática y la audiencia está bien definida; posee una óptima navegabilidad para dispositivos móviles; presenta una ventana gráfica bien configurada, de velocidad moderada; estructura una serie de etiquetas que permite el acceso rápido a temas de interés específico. Se recomienda incluir una página Facebook y Twitter asociada para mejorar la accesibilidad y la difusión. El blog se ha afiliado a otras páginas de interés en la temática: INN, CANIA, Fundación Bengoa, Red-ICEAN. Lo que fortalece su presencia.

\section{CONSIDERACIONES FINALES}

Los blogs, con uso edu-comunicativo, se han extendido intensamente en la web en los últimos años, ya que permiten establecer una comunicación constante y directa entre los actores de la comunidad, a través de sus reflexiones, opiniones y comentarios, expresando sus inquietudes y temas de mayor interés.

Es un recurso -didáctico- versátil al presentar informaciones breves (post) que despiertan el interés del lector llevándolo a investigar y motivándolo a la búsqueda y desarrollo de las ideas expuestas por el autor o por el lector. Apoyando a otros recursos como aulas virtuales, foros de discusión e investigaciones. Aspectos claves en la promoción de la salud integral.

El diseño del blog (Blogger-Google) en educación alimentaria y nutricional, no ha presentado complicaciones y ha dado la oportunidad a los usuarios o lectores, el poder emitir sus comentarios e inquietudes. Esta interactividad, además de la facilidad para su creación, navegabilidad, actualización y administración, es lo que ha influido en el incremento de su presencia dentro de la Internet. Constituyéndose en un recurso en línea idóneo para estudiantes y maestros, sobre temas prioritarios en el campo de la alimentación y nutrición. Se presenta en una forma atractiva y de fácil manejo, se apoya en audio, vídeo, animaciones, presentaciones, documentos, infografías, encuestas, actividades interactivas, entre otros; de fuentes confiables y 
Blog en educación alimentaria y nutricional. Una herramienta de comunicación y educación para promover en salud integral

científicas reconocidas a nivel nacional e internacional, lo que constituye un aporte para la formación integral del individuo.

El blog ha hecho énfasis, durante estos tres años, en la promoción de los valores culturales del hecho alimentario para nuestra sociedad, en los patrones de alimentación más saludables de acuerdo a la edad y condición de vida de la persona, en la revalorización de nuestra cocina tradicional, el impacto de las dietas inadecuadas en la salud, la malnutrición y la aparición de ECNT. Destacando a la alimentación o situación nutricional como una de las múltiples actividades de la vida cotidiana que por su especificidad y evolución debe adquirir un lugar protagónico en la caracterización e interpretación de las dimensiones biológicas, sociales, psicológicas, simbólicas, económicas, religiosas y culturales de las poblaciones humanas y donde lo comunicación, información y educación son los elementos fundamentales.

\section{BIBLIOGRAFÍA}

Alcántara, G. (2008). La definición de salud de la organización Mundial de la Salud y la interdisciplinariedad. Sapiens. Revista Universitaria de Investigación, 9(1), 93107.

De la Cruz, E. (2008). La alimentación. Un acto por repensar, reflexionar y redefinir desde la perspectiva educativa. Integración Universitaria. Revista del instituto Pedagógico de Miranda. 8(2), 23-40. Recuperado de https://www.academia.edu/6942297/LA_ALIMENTACI\%C3\%93.

De la Cruz, E. (2013). Reto y compromiso de la educación alimentaria y nutricional en el siglo XXI. Aportes para su atención permanente desde la perspectiva cultural, socioeconómica y educativa. En: De Tejada y col. Educación Nutricional para un desarrollo sostenible. Caracas: FEDEUPEL.

De la Cruz, E. (2017). La alimentación y nutrición. Referentes de identidad social y patrimonio cultural. Revista CliC. Conocimiento libre y Licenciamiento, 15(8), 129138. Recuperado de https://convite.cenditel.gob.ve/revistaclil(indez.oho/revistaclic/article/view/888.

FAO (2014). Segunda Conferencia Internacional sobre Nutrición. Roma, 19-21 de noviembre. Documento final de la Conferencia. Marco de Acción. Recuperado de http://www.fao.org/3/a-ml542s.pdf

Fundación Bengoa (2010). Faro Nutricional. Recuperado de http://www.fundacionbengoa.org

Instituto Nacional de Nutrición (2008). Sistema de Vigilancia Alimentaria y Nutricional Anuario 2008. Caracas: Instituto Nacional de Nutrición

Mejia, V. (2016). Ventajas del blog. Recuperado de http://tecnologia9854.blogspot.com/2016/05/el-uso-del-blog-como-herramientapara.html

Ministerio del Poder Popular para la salud. (2015). Anuario de Mortalidad 2005-2113. Dirección General de Epidemiología. Dirección de Informática y Estadística de Salud.

Organización Mundial de la Salud (2004). Estrategia Mundial sobre Régimen Alimentario, Actividad Física y Salud. Recuperado de http://www.who. int/dietphysicalactivity/strategy/eb11344/strategy_spanish_web.pdf 
Blog en educación alimentaria y nutricional. Una herramienta de comunicación y educación para promover en salud integral

Osorio, R. (2016). El uso del blog como herramienta. Recuperado de http://usodelblogcomoherramienta.blogspot.com/

Palacio, M. (2016). El uso del blog como herramienta para compartir información... Recuperado de http://tecnooctavo2.blogspot.com/2016/05/uso-del-blog-comoherramienta-para 16.html

Parada, Y. (2014). El uso del blog como recurso didáctico para la enseñanza de computación e informática en el instituto universitario de la policía científica iupolc. Revista Multidisciplinaria Dialógica, 11. Recuperado de http://revistas.upel.edu.ve/index.php/dialogica/article/view/1738

Perea, Rogelia (2002). La educación para la salud. Reto de nuestro tiempo. Educación $X X I, 4,15-40$.

Recuperado de http://espacio.uned.es:8080/fedora/get/bibliuned.EducacionXXIF118F1B1-20B0-6C6D-8F91-9988F26282B4/PDF

Rojas Villada, J. (2016). La tecnología, innovación y evolución. Recuperado de http://latecnologiainnovacioevolucionblogspot.com/2016/05

Sainz, M., López, C. y Van den Boom, A. (2001). Educación para la Salud: Alimentación y Nutrición escolar. Recuperado de http://www.ceapa.es/files/publicaciones/File00117.pdf

Universidad Politécnica de Madrid (2014). Guía sobre el uso educativo de los blogs. Publicación del Gabinete de Tele-Educación. (2014). Recuperado de http://serviciosgate.upm.es/docs/asesoramiento/Blog educativo.pdf 